\title{
Observation of the influences of diosgenin on aging ovarian reserve and function in a mouse model
}

\author{
Mingjie Shen ${ }^{1}$, Cong Qi ${ }^{1^{*}}$, Yan-Ping Kuang ${ }^{2 *}$, Yang Yang ${ }^{3}$, Qi-Feng Lyu ${ }^{2}$, Hui Long ${ }^{2}$, Zhi-Guang Yan ${ }^{2}$ \\ and Ying-Yu Lu'
}

\begin{abstract}
Background: The aim of this study was to investigate the impact of diosgenin, an important monomer of sapogenins in yams, on ovarian reserve in a natural aging mice model.

Study design: This randomized controlled trial included 60 9-month-old C57 naturally aging female mice. Twentyone mice were assigned to the dio group and were fed a single dose of diosgenin ( $200 \mathrm{mg} / \mathrm{kg} /$ day) suspended in $0.3 \%$ CMC. Twenty mice were assigned to the DHEA group and were fed a single dose of DHEA $(1.25 \mathrm{mg} / \mathrm{kg} /$ day $)$ suspended in $0.3 \%$ CMC. The remaining 20 mice were assigned to the old control group and were fed a single dose of $0.3 \%$ CMC. Three months later, the reproductive performance of these female mice was determined by evaluating ovarian follicles and oocyte number and quality in IVF and comparing age-matched and young controls. The impact of NOBOX, GDF9 and BMP15 mRNA expression was also evaluated.
\end{abstract}

Results: Diosgenin improves ovarian reserve in naturally aging mice in terms of increasing the number of primary follicles $(P<0.05)$ and serum levels of AMH $(P<0.05)$.

Conclusions: Diosgenin could counteract age-associated ovarian dysfunction by improving the ovarian reserve in a natural aging mice model.

Keywords: Diosgenin, Ovarian reserve, Aging, Mouse model

\section{Background}

Many women in the workforce postpone their childbearing, but their aging ovaries constitute a robust negative factor when they attempt to conceive [1]. Age-associated infertility has also been a great challenge to doctors using assisted-reproductive technologies. It has been reported that age is the strongest predictor of ovarian response and pregnancy rate [2]. Most evidences support the concept that women are born with a fixed number of oocytes

\footnotetext{
*Correspondence: qi_congdr@163.com; kuang_yp@126.com; kuang_ yp5897@126.com

${ }^{1}$ Department of Gynecology and Obstetrics, Shu Guang Hospital Affiliated to Shanghai University of Traditional Chinese Medicine, Road Zhangheng No. 528, Pu Dong District, Shanghai 201203, China

${ }^{2}$ Department of Assisted Reproduction, Shanghai Ninth People's Hospital Affiliated to Shanghai Jiao Tong University of Medicine, Road Zhizaoju No. 639 Huangpu District, Shanghai 200000, China

Full list of author information is available at the end of the article
}

that cannot regenerate and are depleted with age [3]. However, in contrast, few studies have suggested the presence of germ stem cells that could potentially replace lost follicles $[4,5]$. It is known that the ovarian primordial follicle reserve is established during fetal development and that after birth the primordial follicle pool is continuously activated, while the rest of the pool remains quiescent for years or even decades until menopause [6]. With the remarkable decline in ovarian reserve, women by the age of 30 years retain only $12 \%$ of their ovarian reserve; and by the age of 40 years only $3 \%$ [7]. The extremely low pregnancy rate of women over the age of 40 years results from the decline in primordial follicle numbers. This parallels the decrease in healthy growing small antral follicles (an important dynamic reserve for ovulation) and the deterioration of oocyte quality [8]. 
Since the significant diminution in the ovarian reserve is a physiologic and anatomic fact that older women have to face, there is a need for greater attention on efficiency related to follicular development. Data indicate that the overwhelming majority of follicles undergo atresia at a relatively early stage of follicular development. Improving the efficiency of follicular development and preventing more primordial follicles from attaining the fate of atresia is the strategy we can use, in principle, to protect against ovarian aging and thereby prolong the reproductive life span. This is the current focus of our study.

The molecular control of oogenesis is complicated. A vast number of ovarian factors regulate this process including members of the transforming growth factorbeta (TGF- $\beta$ ) family, which control the growth and differentiation of somatic and germ cells. Growth differentiation factor-9 (GDF-9) and bone morphogenetic protein-15 (BMP-15) of the TGF $\beta$ family are well-known ovarian factors that regulate the process of follicular development and are secreted by oocytes [9]. With respect to the activation and suppression of primordial follicles, the PTEN/PI3k pathway plays a vital role [10, 11]. A recent study has indicated that newborn ovary homeobox-encoding gene (NOBOX), one of the oocytespecific transcription factors, is an important player in the activation of primordial follicles and the transition to primary follicles. Without NOBOX, the majority of ovarian follicles are arrested at the primordial stage, and oocytes degenerate and do not develop beyond singlelayered cuboidal primary follicles. NOBOX expression can also influence other important oocyte transcripts such as GDF-9, BMP-15 and Oct4 that occupy roles at different follicular stages [12].

Diosgenin ([25R]-5-spirosten-3 $\beta$-ol) is a naturally occurring steroidal saponin that is present in a variety of plants including Dioscorea species, fenugreek and Costus speciosus [13]. Diosgenin has been initially acknowledged to be the starting material for the synthesis of a number of steroid hormones [14] and has now been reported to exert antiproliferative and proapoptotic actions on rheumatoid arthritis synoviocytes [15]. In addition, diosgenin exhibits other biological activities such as anticancer activity [16-18], antiviral activity [19] and antiinflammatory activity [20]. Diosgenin even shows potentially practical applications in the clinical treatment of heart disease [21]. However, diosgenin has never been reported to demonstrate actions with respect to improving ovarian function. Thus far, as an important monomer in yams (which has been acknowledged to improve women's ovarian function in traditional Chinese medicine, and is included by millions of Chinese women in their diets), there is the distinct possibility that diosgenin might improve ovarian function.
In this study, we hypothesize that diosgenin counteracts age-associated ovarian dysfunction and improves the ovarian reserve in a mouse model of reproductive aging. We performed a 3-month administration of diosgenin in mice, to test the effects of diosgenin on improving overall reproductive function.

\section{Methods}

\section{Animals and treatments}

Female C57 mice were purchased at 9 months of age from the Vital River Laboratory Animal Technology Co., Ltd. The animals were housed under 12:12-h light-dark cycle conditions in a specific pathogen-free animal facility located at the Experimental Animal Center of Shanghai University of Traditional Chinese Medicine, China. The protocol of this study was approved by the Institutional Animal Committee. Mice were randomly divided into three groups: old control group, mice were fed $0.3 \%$ sodium carboxymethyl cellulose (CMC; purchased from Yuanye Bio-Technology Co., Ltd., Shanghai, China); DHEA group, mice were fed $1.25 \mathrm{mg} / \mathrm{kg} /$ day of dehydroepiandrosterone (DHEA; purchased from General Nutrition Center Inc., Pittsburgh, PA, USA), suspended in 0.3\% CMC; dio group, mice were fed diosgenin $(99 \%$ purity; purchased from Yuanye Bio-Technology Co., Ltd., Shanghai, China) at $200 \mathrm{mg} / \mathrm{kg} /$ day, suspended in $0.3 \%$ CMC. These treatments were intragastrically administered in each group once daily. Following treatment with diosgenin or DHEA for 3 months, some of the mice were randomly chosen to assess the ovarian reserve, ovarian response and oocyte quality with in vitro fertilization (IVF); while the remaining mice were used for breeding and evaluating litter size. Other mice were used to assess the expression of genes related to follicular development. Young mice at the age of 2-3 months served as controls (young control group) and were purchased from the Vital River Laboratory Animal Technology Co., Ltd.

\section{Ovarian serial sectioning and quantification of follicle counts}

Ovaries were randomly collected from mice in the different groups (dio, DHEA, old control and young control groups). After immersion in 10\% neutral-buffered formalin for at least 1 week, the tissues were dehydrated and embedded in paraffin wax and serially sectioned. Serial sections $(5 \mu \mathrm{m})$ of each ovary were orderly aligned on glass microscope slides, stained with hematoxylin and eosin Y, and analyzed for the numbers of follicles at four different developmental stages using every fifth section with a random start in the first five sections. The total number of follicles per ovary was calculated by combining the counts in every fifth section throughout each entire ovary. 
The follicles were categorized as primordial, primary, secondary, antral or pre-ovulatory, according to a previous study [22]. Follicles were classified as primordial if these contained an oocyte surrounded by a single layer of squamous granulosa cells, and were classified as primary if these were surrounded by a single layer of cuboidal granulosa cells. Secondary follicles were identified as having more than one layer of granulosa cells with no visible antrum. Antral follicles had small areas of follicular fluid (antrum), while pre-ovulation follicles had a single large antral space and cumulus oophorus.

\section{Anti-Mullerian hormone (AMH) measurements}

A mouse AMH kit (US Biological Life Sciences, 23452, USA) was used to determine plasma AMH levels through enzyme-linked immunosorbent assay (ELISA). The analytical sensitivity of the kit was $0.05 \mathrm{ng} / \mathrm{mL}$, and the standard curve spanned the range from 0.1 to $40 \mathrm{ng} / \mathrm{mL}$.

\section{Oocyte retrieval}

Female mice from the different groups were superovulated by injecting $10 \mathrm{IU}$ of equine chorionic gonadotropin (eCG), followed by the administration of $10 \mathrm{IU}$ of human chorionic gonadotropin (hCG) after $48 \mathrm{~h}$. Female mice were humanely killed $\left(\mathrm{CO}_{2}\right.$ overdose $) 16 \mathrm{~h}$ after hCG injection and the oviducts were collected. The cumulusoocyte complexes (COCs) were released from the ampullar region of each oviduct by puncturing the oviduct with a 28-gauge needle affixed to a $1-\mathrm{mL}$ syringe, and these were collected by flushing the oviducts with human tubal fluid (HTF; EmbryoMax ${ }^{\circledR}$, Millipore, USA). COCs were transferred and cultured in KSOM medium (Millipore, USA) with $5 \% \mathrm{CO}_{2}$ at $37^{\circ} \mathrm{C}$ and stored until use.

\section{IVF and embryo culture}

To obtain sperm for IVF, 12-week-old male ICR mice were euthanized by cervical dislocation and epididymides were collected by dissection. Then, the epididymides were placed in the central well of an IVF dish with HTF medium. After making five to seven longitudinal cuts on each epididymis using a needle-affixed syringe, the epididymides were incubated for $20 \mathrm{~min}$ at $37^{\circ} \mathrm{C}$ with $5 \% \mathrm{CO}_{2}$ in compressed air to allow for sperm dispersion. The sperm suspensions were incubated for $1 \mathrm{~h}$ at $37^{\circ} \mathrm{C}$ with $5 \% \mathrm{CO}_{2}$ air to allow for capacitation. For IVF, MII oocytes were inseminated with $2 \times 10^{4}$ sperms in a droplet of $150 \mu \mathrm{L}$ of HTF medium for $4.5 \mathrm{~h}$. The fertilized oocytes were subsequently cultured in a drop of $20 \mu \mathrm{L}$ of KOSM medium at $37{ }^{\circ} \mathrm{C}$ with $5 \% \mathrm{CO}_{2}$ in compressed air and high humidity. The development of the fertilized oocytes was monitored under an inverted microscope (Motic AE2000TRI) for the formation of two-cell-, four-cell-, morula-, and blastocyststage embryos at various intervals for up to 5 days.

\section{Gene expression by real-time PCR}

Ovaries were lysed using TRIzol reagent (Invitrogen, Carlsbad, CA, USA) and DNA was reverse transcribed. The mRNA levels of three important genes related to follicle growth were measured by real-time PCR (RT-PCR) using $\mathrm{iQSYBR}$ green reagent (Qiagen, Valencia, CA, USA) and a Continuous Fluorescence Detection System (M) Research Inc., Waltham, MA, USA). The mRNA expression was normalized to that of hypoxanthine-guanine phosphoribosyltransferase (HGPRT) for each sample, and the fold changes for each gene were calculated against those in normal mice. The details are shown in "Statistical analysis".

\section{Statistical analysis}

Experimental data were presented as mean \pm standard deviation (SD). Data were obtained from three independent experiments, with three replicates per experiment. Data were evaluated by one-way analysis of variance (ANOVA) with the Tukey HSD post hoc test for comparisons between groups. $P<0.05$ was considered to be statistically significant.

\section{Diosgenin increases primary follicle numbers}

Young C57 females exhibited a large number of primary and primordial follicles, as well as secondary, antral and mature follicles. At the same time, the number of follicles was remarkably reduced in every category of aging female mice (old control group). Age-matched female mice treated with diosgenin exhibited more primary and primordial follicles, compared with aging controls. Although the number of primary follicles in the dio group was statistically greater than that in the old control group, the difference in the number of primordial follicles was not statistically significant (albeit a tendency for an increase was observed). However, diosgenin did not increase the number of growing or pre-ovulatory follicles after 3 months of treatment. Furthermore, the number of atretic follicles did not differ between the old control and the dio groups (Figs. 1 and 2, Table 1).

\section{Diosgenin increased AMH serum levels}

Anti-Mullerian hormone serum levels in aging mice (old control group) were significantly decreased compared with the young control group, while AMH levels were markedly higher in aging mice treated with diosgenin (dio group) compared with age-matched mice in the old control group and the mice treated with DHEA (Fig. 3).

\section{Diosgenin tends to increase the number of oocytes retrieved and improves the fertilization rate in IVF}

The number of oocytes retrieved, MII oocytes and fertilized oocytes in aging mice treated with diosgenin tended 


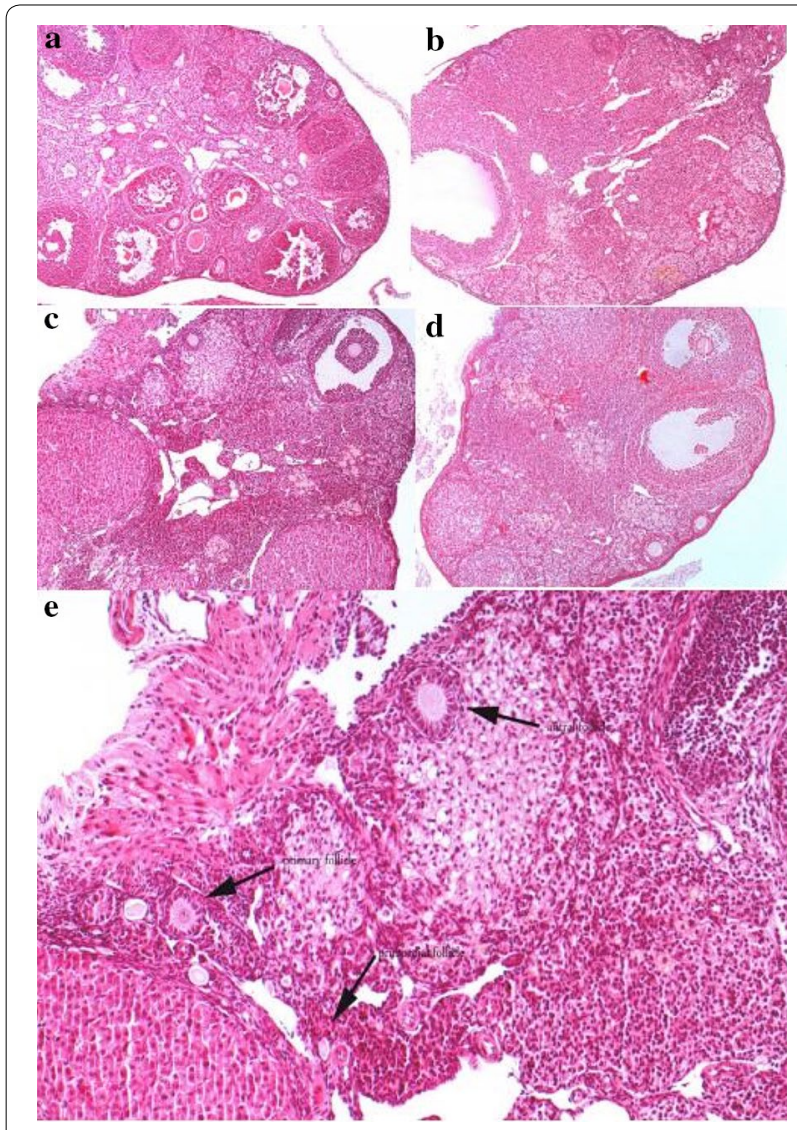

Fig. 1 Typical photomicrographs $(\times 100)$ of the mice in the young control group (a), old control group (b), dio group (c) and DHEA group (d), and the details of the dio group (e)

to increase, compared with the old control and DHEA groups, but the difference was not statistically significant (Table 2).

\section{Diosgenin influences the gene expression of NOBOX and GDF-9}

The expression of NOBOX, GDF-9 and BMP-15 decreased in aging mouse (old control group) ovaries compared with the young mice $(P<0.05)$. However, in ovaries from aging mice treated with diosgenin, NOBOX and GDF-9 gene expression revealed a tendency to increase; but the increase was not significant. This was also the case for aging mice treated with DHEA with regard to the expression of GDF-9. Neither diosgenin nor DHEA influenced the expression of BMP-15 (Fig. 4).

\section{Discussion}

Our results indicate that diosgenin can increase the number of primary follicles, and a tendency for the number of primordial follicles to increase was observed, thereby improving the ovarian reserve in aging mice. Diosgenin revealed a tendency to increase oocyte retrieval and

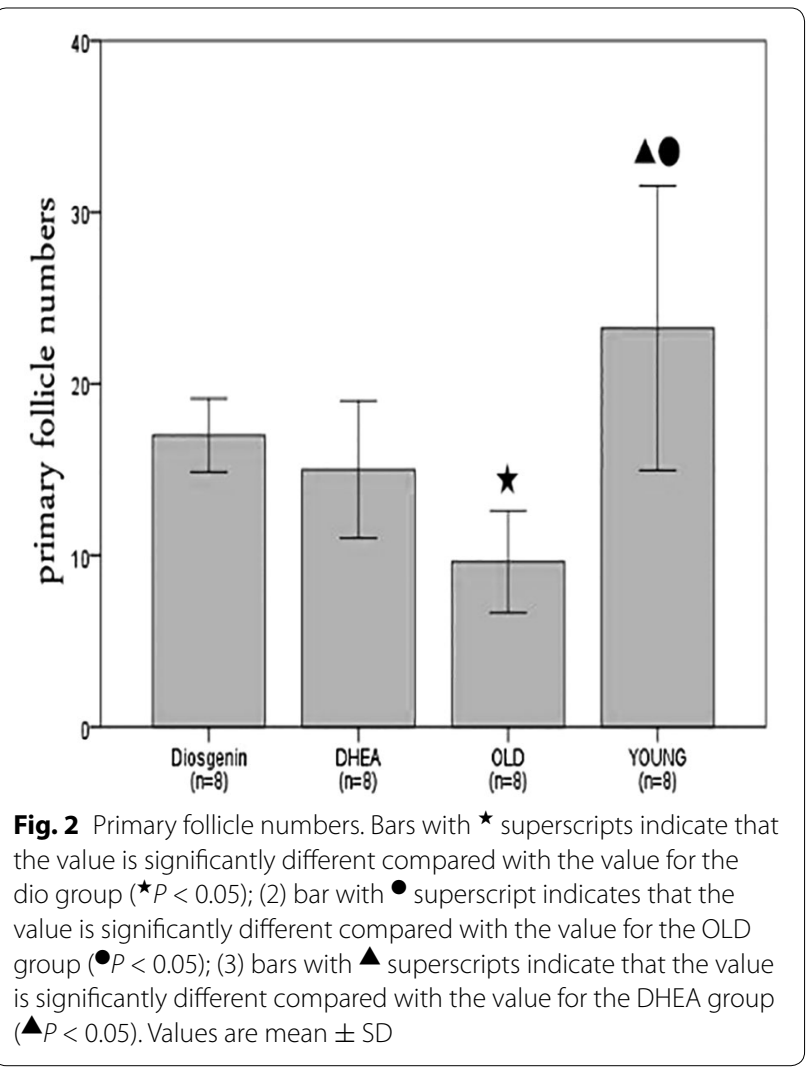

fertilization rate in vitro. This suggests that diosgenin can slow ovarian aging and improve the response to gonadotropin by aging ovaries in IVF by replenishing the reduction in the follicular pool.

According to classical gynecologic theory, female mammals are born with a fixed number of oocytes that continuously reduces until these animals are no longer able to conceive [23]. The potential presence of ovarian stem cells remains controversial, and most scholars unequivocally state that oocytes in female mammals cannot be renewed.

In today's society, more women continue to delay their childbirth; therefore, when a woman first wishes to conceive at the age of 30 or even 40 years, the reduction in oocyte quality and quantity can cause problematic issues. In terms of classic gynecologic theory, the tendency for the decline in fertility is inevitable. However, this does not mean that we have no ability to delay ovarian aging. Our results revealed that diosgenin treatment contributed to the improvement in the ovarian reserve of naturally aging mice by increasing the number of primary follicles, when compared with aging controls.

Anti-Mullerian hormone is produced by granulosa cells of developing pre-antral and small antral follicles [24]. Serum AMH levels are widely used to estimate the size of the ovarian reserve and are regarded as a sensitive 
Table 1 Follicle counts of ovarian specimens from diosgenin, DHEA, old and young groups

\begin{tabular}{|c|c|c|c|c|c|c|c|}
\hline Group & $n$ & Primordial & Primary & Secondary & Antral & Pre-ovulation & Atretic \\
\hline Diosgenin & 8 & $18.63 \pm 10.06$ & $17.00 \pm 2.14$ & $6.63 \pm 2.26$ & $6.75 \pm 2.92$ & $2.13 \pm 2.64$ & $1.88 \pm 2.23$ \\
\hline DHEA & 8 & $10.50 \pm 3.38$ & $15.00 \pm 4.00$ & $8.50 \pm 3.89$ & $7.25 \pm 2.82$ & $0.50 \pm 0.53$ & $1.13 \pm 1.36$ \\
\hline Old & 8 & $8.88 \pm 4.88$ & $9.63 \pm 2.97^{\star}$ & $5.88 \pm 2.64$ & $6.25 \pm 3.01$ & $1.50 \pm 1.93$ & $3.63 \pm 1.92 \boldsymbol{\Delta}$ \\
\hline Young & 8 & $27.38 \pm 8.62 \boldsymbol{\Delta} \bullet$ & $23.25 \pm 8.29 \boldsymbol{\bullet}$ & $30.25 \pm 8.92 \star \boldsymbol{\Delta} \bullet$ & $65.50 \pm 17.39 \star \Delta \bullet$ & $10.63 \pm 3.46^{\star} \boldsymbol{\Delta} \bullet$ & $0.50 \pm 0.76^{\bullet}$ \\
\hline F & & 10.979 & 10.321 & 40.821 & 84.182 & 30.431 & 5.272 \\
\hline$P$ & & $<0.001$ & $<0.001$ & $<0.001$ & $<0.001$ & $<0.001$ & 0.005 \\
\hline
\end{tabular}

(1) Values with ${ }^{\star}$ superscripts are significantly different compared with the value for the dio group $\left({ }^{\star} P<0.05\right)$; (2) values with $\bullet$ superscript are significantly different

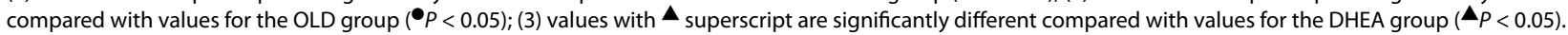
Values are mean \pm SD

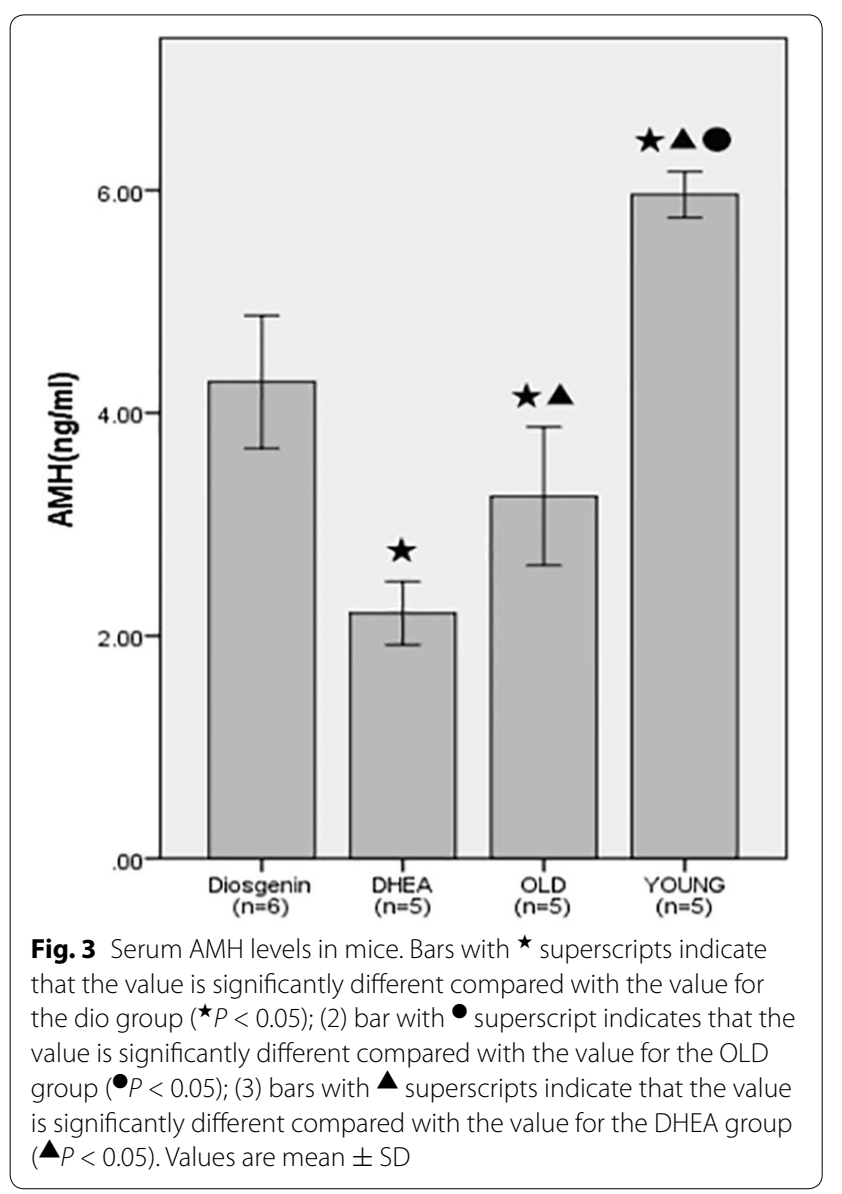

indicator of ovarian function in the clinic [25]. In our study, serum AMH levels, together with the quantification of follicle numbers, indicated that the ovarian reserve in aged mice could be improved to some extent. In addition, IVF results revealed that diosgenin tended to increase the number of oocytes retrieved, MII oocytes and fertilized oocytes in aging mice, although our interpretations were limited by the small sample size. The increased number of MII oocytes and fertilized oocytes in the dio group, compared to the old control group, indicates that diosgenin could improve both the number and quality of oocytes. Therefore, diosgenin appeared to protect against ovarian aging and contributed to the improvement in IVF results in aging mice.

Primordial follicles constitute the total reservoir of germ cells available during the entire female reproductive life span. Despite this, the mechanism(s) for the formation and activation of primordial follicles are not fully understood, although GDF9, BMP15 and NOBOX are known to be involved in these processes.

GDF9 and BMP15 are important members of the TGF- $\beta$ family and are produced exclusively by growing oocytes in the ovary, playing a vital role in regulating the development of oocytes and proliferation and differentiation of ovarian granulosa cells. In GDF9-null mice, folliculogenesis does not progress beyond the primary stage [26], while BMP15-null mice have impaired oocyte maturation that leads to infertility [27]. In our study, GDF9 and BMP15 were significantly different between the young and old control groups, which indicate that the reduced ovarian reserve observed with decreasing preantral follicle count was associated with the reduction in the expression of GDF9 and BMP15 in aging ovaries. NOBOX is an oocyte-specific homeobox gene expressed in germ cell cysts, as well as in primordial and growing oocytes. The proper expression of NOBOX is crucial for ovarian development in both mice and humans [28-36]. Our data also confirmed these concepts. The reduced expression of NOBOX in the ovaries was commensurate with ovarian aging and the reduction in the ovarian reserve.

Our results revealed that there were increasing numbers of primary follicles and a growth trend of primordial follicles in the dio group, compared with the old control group. Furthermore, this suggests that diosgenin appears to stimulate the formation and activation of primordial follicles. Consequently, we hypothesized that the ovarian reserve could be improved in old mice treated by 
Table 2 Effects of oocyte retrieval and fertilization in vitro (IVF)

\begin{tabular}{llllll}
\hline Group & $\boldsymbol{n}$ & No. of oocytes per mouse & No. of MIl oocytes per mouse & No. of fertilized oocytes per mouse & Fertilization rate \\
\hline Dio & 5 & $2.80 \pm 2.59$ & $1.80 \pm 1.64$ & $1.60 \pm 1.52$ & $0.73 \pm 0.43$ \\
DHEA & 5 & $1.00 \pm 1.22$ & $0.80 \pm 0.84$ & $0.40 \pm 0.55$ & $0.30 \pm 0.45$ \\
Old & 5 & $0.80 \pm 1.10$ & $0.60 \pm 0.89$ & $0.40 \pm 0.55$ & $0.30 \pm 0.45$ \\
Young & 5 & $12.00 \pm 2.74 \star \boldsymbol{\Delta} \bullet$ & $9.80 \pm 2.86 \star \boldsymbol{\Delta} \bullet$ & $9.60 \pm 2.61 \star \boldsymbol{\bullet} \bullet$ & $0.98 \pm 0.03$ \\
F & & 33.369 & 31.199 & 40.577 & 3.887 \\
$P$ & & $<0.001$ & $<0.001$ & $<0.001$ & 0.029 \\
\hline
\end{tabular}

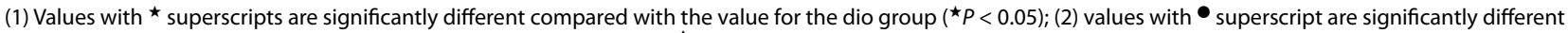
compared with the value for the Old group $(\bullet<0.05)$; (3) values with $\mathbf{\Lambda}$ superscript are significantly different compared with the value for the DHEA group $(\mathbf{\Delta} P<0.05)$. Values are mean \pm SD
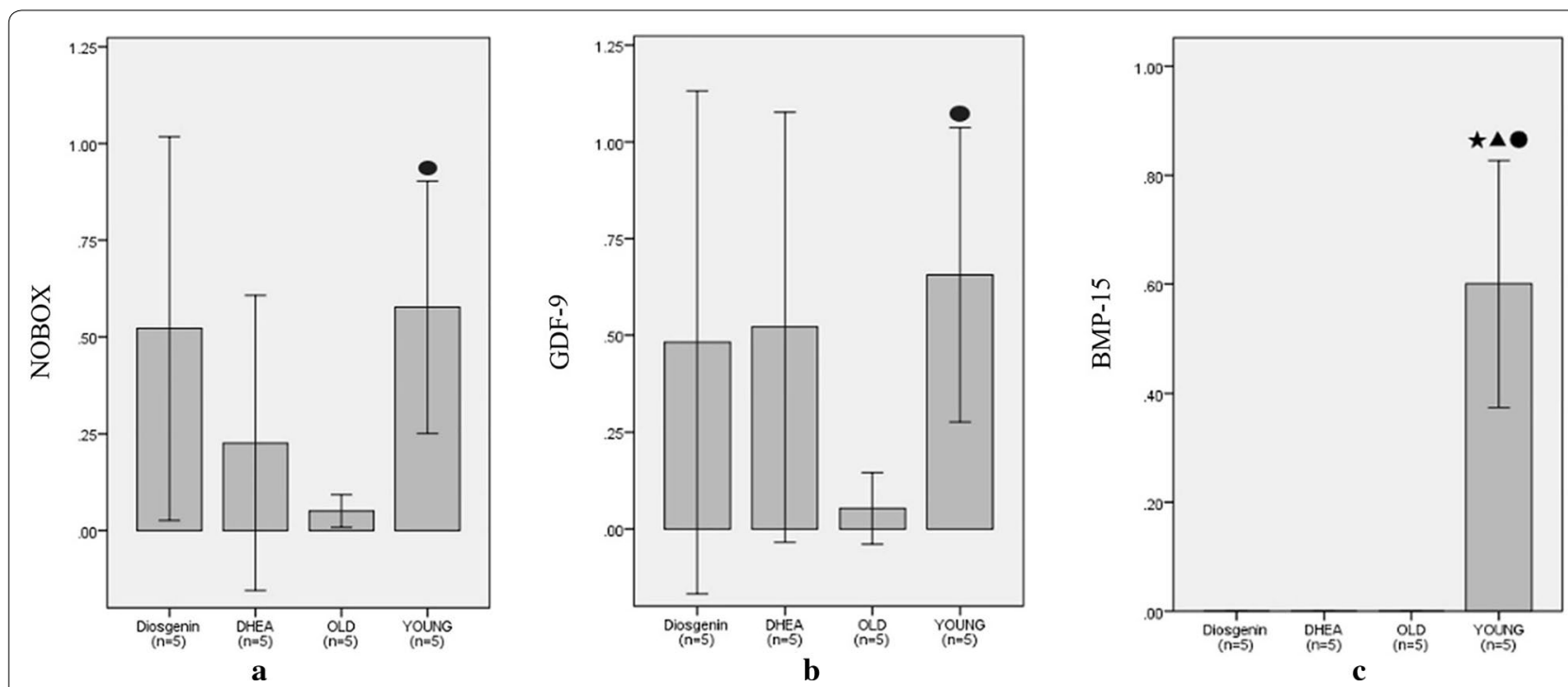

Fig. 4 Three oocyte-specific gene expression in ovaries. $\mathbf{a}$ The relative mRNA levels of NOBOX, $\mathbf{b}$ the relative mRNA levels of GDF-9, and $\mathbf{c}$ the relative mRNA levels of BMP-15. (1) Bars with * superscripts indicate that the value is significantly different compared with the value for the dio group $(\star P<0.05)$; (2) bars with $\bullet$ superscript indicates that the value is significantly different compared with the value for the OLD group $(\bullet P<0.05)$;

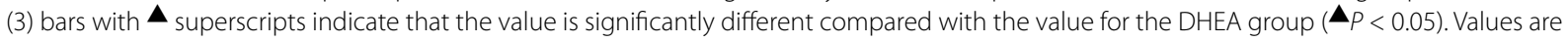
mean \pm SD

diosgenin. NOBOX was expressed in oocytes, and GDF9 was expressed in granulosa cells in growing follicles. Due to increasing numbers of primary follicles and the growth trend of primordial follicles in the dio group, the elevated level of NOBOX and GDF9 was understandable. Interestingly, the expression of GDF9 was also increased in mice in the DHEA group. Despite this, there was a slight non-significant increase in the number of primordial, primary and secondary follicles in the DHEA group. DHEA appeared to increase the number of pre-antral follicles to some extent, but did not enhance the retrieval of MII oocytes or promote the quality of oocytes in our study. This might be related to the reduced expression of NOBOX and increased expression of GDF9 in the DHEA group.

\section{Conclusions}

The present study supports our hypothesis that diosgenin increases the number of primary follicles, leading to the promotion of ovarian reserve in a naturally aging mouse model. Although our research shows that diosgenin benefits ovarian reserve in aging mice, data in this mouse model may not be directly extrapolatable to human ovarian reserve. Hence, more extensive research is needed if any clinic trials are to be attempted.

\section{Authors' contributions}

MJS and CQ acquired data. YPK and YY drafted the manuscript. HL, YYL and ZGY contributed substantially to its revision. MJS, QFL and CQ take responsibility for the paper as a whole. All authors read and approved the final manuscript. 


\section{Author details}

${ }^{1}$ Department of Gynecology and Obstetrics, Shu Guang Hospital Affiliated to Shanghai University of Traditional Chinese Medicine, Road Zhangheng No. 528, Pu Dong District, Shanghai 201203, China. ${ }^{2}$ Department of Assisted Reproduction, Shanghai Ninth People's Hospital Affiliated to Shanghai Jiao Tong University of Medicine, Road Zhizaoju No. 639 Huangpu District, Shanghai 200000 , China. ${ }^{3}$ Laboratory of Immunology and Virology, Experiment Center for Science and Technology, Shanghai University of Traditional Chinese Medicine, Shanghai, China.

\section{Acknowledgements and funding}

This work was supported by the National Natural Science Foundation of China (Grant No. 81403424), Shanghai Natural Science Foundation (Grant No. 14ZR1442100), and Shanghai Three Years Action Plan of Traditional Chinese Medicine-Innovation construction of Traditional Chinese Medicine (Grant No. ZY3-CCCX-3-3007).

We thank LetPub (http://www.letpub.com) for its linguistic assistance during the preparation of this manuscript.

We are particularly grateful to all the people who have given us help for our article.

\section{Competing interests}

The authors declare that they have no competing interests.

\section{Availability of data and materials}

All of the data and materials are available.

\section{Consent for publication}

All participants signed a document of informed consent.

\section{Ethics approval and consent to participate}

This study was approved by the Ethical Committee of our hospital.

\section{Publisher's Note}

Springer Nature remains neutral with regard to jurisdictional claims in published maps and institutional affiliations.

Received: 27 February 2017 Accepted: 4 October 2017 Published online: 18 October 2017

\section{References}

1. Mills M, Rindfuss RR, McDonald P, et al. Why do people postpone parenthood? Reasons and social policy incentives. Hum Reprod Update. 2011;17:848-60.

2. Broer SL, Disseldorp JV, Broeze KA, et al. Added value of ovarian reserve testing on patient characteristics in the prediction of ovarian response and ongoing pregnancy: an individual patient data approach. Hum Reprod Update. 2013;19:26-36.

3. Hanna CB, Hennehold JD. Ovarian germline stem cells: an unlimited source of oocytes. Fertil Steril. 2014;101:20-30.

4. Johnson J, Canning J, Kaneko T, Pru JK, Tilly JL. Germline stem cells and follicular renewal in the postnatal mammalian ovary. Nature. 2004;428:145-50.

5. Johnson J, Bagley J, Skaznik-Wikiel M, et al. Oocyte generation in adult mammalian ovaries by putative germ cells in bone marrow and peripheral blood. Cell. 2005:122:303-15.

6. McGee EA, Hsueh AJ. Initial and cyclic recruitment of ovarian follicles. Endocr Rev. 2000;21:200-14.

7. Wallace WH, Kelsey TW. Human ovarian reserve from conception to the menopause. PLOS ONE. 2010;5:1-7.

8. Broekmans FJ, Soules MR, Fauser BC. Ovarian aging: mechanisms and clinical consequences. Endocr Rev. 2009;30:465-93.

9. Pangas SA. Regulation of the ovarian reserve by members of the transforming growth factor beta family. Mol Reprod Dev. 2012;79:666-79.

10. Reddy P, Shen L, Ren C, Boman K, Lundin E Ottander U, Lindgren P, Liu YX, Sun QY, Liu K. Activation of Akt (PKB) and suppression of FKHRL1 in mouse and rat oocytes by stem cell factor during follicular activation and development. Dev Biol. 2005;281:160-70.

11. Cantley LC. The phosphoinositide 3-kinase pathway. Science. 2002:296:1655-7.

12. Rajkovic A, Pangas SA, Ballow D, et al. NOBOX deficiency disrupts early folliculogenesis and oocyte-specific gene expression. Science. 2004;305:1157-9.

13. Sautour M, Mitaine-Offer AC, Miyamoto T, et al. Antifungal steroid saponins from Dioscorea cayenensis. Planta Med. 2004;70:90-2.

14. Scott A, Higdon K, Tucci M, et al. The prevention of osteoporotic progression by means of steroid loaded TCPL drug delivery systems. Biomed Sci Instrum. 2001;37:13-8.

15. Liagre B, Vergne-Salle P, Corbiere C, et al. Diosgenin, a plant steroid, induces apoptosis in human rheumatoid arthritis synoviocytes with cyclooxygenase-2 overexpression. Arthritis Res Ther. 2004:6:373-83.

16. Raju J, Mehta R. Cancer chemopreventive and therapeutic effects of diosgenin, a food saponin. Nutr Cancer. 2009;61:27-35.

17. Lepage C, Léger DY, Bertrand J, et al. Diosgenin induces death receptor-5 through activation of p38 pathway and promotes TRAlL-induced apoptosis in colon cancer cells. Cancer Lett. 2011;301:193-202.

18. Chen PS, Shih YH, Huang HC, et al. Diosgenin, a steroidal saponin, inhibits migration and invasion of human prostate cancer PC-3 cells by reducing matrix metalloproteinases expression. PLoS ONE. 2011;6:e20164.

19. Wang YJ, Pan KL, Hsieh TC, et al. Diosgenin, a plant derived sapogenin, exhibits antiviral activity in vitro against hepatitis C virus. J Nat Prod. 2011;74:580-4.

20. Jung $\mathrm{DH}$, Park HJ, Byun $\mathrm{HE}$, et al. Diosgenin inhibits macrophage-derived inflammatory mediators through downregulation of CK2, JNK, NF-KB and AP-1 activation. Int Immunopharmacol. 2010;10:1047-54.

21. Liu K, Zhao W, Gao X, et al. Diosgenin ameliorates palmitate-induced endothelial dysfunction and insulin resistance via blocking IKK $\beta$ and IRS-1 pathways. Atherosclerosis. 2012;223:350-8.

22. Myers M, Britt KL, Wreford NG, et al. Methods for quantifying follicular numbers within the mouse ovary. Reproduction. 2004;127:569-80.

23. Faddy MJ, Gosden RG, Gougeon A, et al. Accelerated disappearance of ovarian follicles in mid-life: implications for forecasting menopause. Hum Reprod. 1992;7:1342-6.

24. Jeppesen JV, Anderson RA, Kelsey TW, et al. Which follicles make the most anti-Mullerian hormone in humans? Evidence for an abrupt decline in $\mathrm{AMH}$ production at the time of follicle selection. Mol Hum Reprod. 2013:19:519-27.

25. Visser JA, de Jong FH, Laven JS, et al. Anti-Mullerian hormone: a new marker for ovarian function. Reproduction. 2006;131:1-9.

26. Dong J, Albertini DF, Nishimori K, et al. Growth differentiation factor-9 is required during early ovarian folliculogenesis. Nature. 1996;383:531-5.

27. Yan C, Wang P, DeMayo J, et al. Synergistic roles of bone morphogenetic protein 15 and growth differentiation factor 9 in ovarian function. Mol Endocrinol. 2001;15:854-66.

28. Choi Y, Rajkovic A. Genetics of early mammalian folliculogenesis. Cell Mol Life Sci. 2006:63:579-90.

29. Choi Y, Rajkovic A. Characterization of NOBOX DNA binding specificity and its regulation of Gdf9 and Pou5f1 promoters. J Biol Chem. 2006;281:35747-56

30. Choi Y, Qin Y, Berger MF, et al. A microarray analyses of newborn mouse ovaries lacking NOBOX. Biol Reprod. 2007;77:312-9.

31. Huntriss J, Hinkins M, Picton HM. cDNA cloning and expression of the human NOBOX gene in oocytes and ovarian follicles. Mol Hum Reprod. 2006;12:283-9.

32. Qin Y, Choi Y, Zhao H, et al. NOBOX homeobox mutation causes premature ovarian failure. Am J Hum Genet. 2007:81:576-81.

33. Qin Y, Shi Y, Zhao Y, et al. Mutation analysis of NOBOX homeodomain in Chinese women with premature ovarian failure. Fertil Steril. 2009:91:1507-9.

34. Simpson JL. Genetic and phenotypic heterogeneity in ovarian failure: overview of selected candidate genes. Ann NY Acad Sci. 2008;1135:146-54.

35. Suzumori N, Yan C, Matzuk MM, et al. NOBOX is a homeobox-encoding gene preferentially expressed in primordial and growing oocytes. Mech Dev. 2002;111:137-41.

36. Suzumori N, Pangas SA, Rajkovic A. Candidate genes for premature ovarian failure. Curr Med Chem. 2007;14:353-7. 